\title{
Activity of ceftolozane/tazobactam against Gram-negative isolates from patients with lower respiratory tract infections - SMART United States 2018-2019
}

James A. Karlowsky ${ }^{1,2}$, Sibylle H. Lob ${ }^{1 *}$, Katherine Young ${ }^{3}$, Mary R. Motyl $\left.\right|^{3}$ and Daniel F. Sahm ${ }^{1}$

\begin{abstract}
Background: Ceftolozane/tazobactam (C/T) is approved in 70 countries, including the United States, for the treatment of patients with hospital-acquired and ventilator-associated bacterial pneumonia caused by susceptible Gram-negative pathogens. $C / T$ is of particular importance as an agent for the treatment of multidrug-resistant (MDR) Pseudomonas aeruginosa infections. The current study summarizes 2018-2019 data from the United States on lower respiratory tract isolates of Gram-negative bacilli from the SMART global surveillance program. The CLSI reference broth microdilution method was used to determine in vitro susceptibility of $C / T$ and comparators against isolates of $P$. aeruginosa and Enterobacterales.

Results: $C / T$ inhibited $96.0 \%$ of $P$. aeruginosa $(n=1237)$ at its susceptible MIC breakpoint $(\leq 4 \mu \mathrm{g} / \mathrm{ml})$, including $>85 \%$ of meropenem-nonsusceptible and piperacillin/tazobactam (P/T)-nonsusceptible isolates and 76.2\% of MDR isolates. Comparator agents demonstrated lower activity than C/T against $P$. aeruginosa: meropenem (74.8\% susceptible), cefepime (79.2\%), ceftazidime (78.5\%), P/T (74.4\%), and levofloxacin (63.1\%). C/T was equally active against ICU (96.0\% susceptible) and non-ICU (96.7\%) isolates of P. aeruginosa. C/T inhibited $91.8 \%$ of Enterobacterales $(n=1938)$ at its susceptible MIC breakpoint ( $\leq 2 \mathrm{\mu g} / \mathrm{ml}) ; 89.5 \%$ of isolates were susceptible to cefepime and $88.0 \%$ susceptible to $P / T$. 67.1 and $86.5 \%$ of extended-spectrum $\beta$-lactamase (ESBL) screen-positive isolates of Klebsiella pneumoniae $(n=85)$ and Escherichia coli $(n=74)$ and $49.6 \%$ of MDR Enterobacterales were susceptible to $C / T$. $C / T$ was equally active against ICU (91.3\% susceptible) and non-ICU (92.6\%) Enterobacterales isolates.
\end{abstract}

Conclusion: Data from the current study support the use of $\mathrm{C} / \mathrm{T}$ as an important treatment option for lower respiratory tract infections including those caused by MDR P. aeruginosa.

Keywords: Ceftolozane/tazobactam, Enterobacterales, Pseudomonas aeruginosa, United states, SMART, Surveillance

\footnotetext{
* Correspondence: shlob@ihma.com

'IHMA, 2122 Palmer Drive, Schaumburg, IL 60173, USA

Full list of author information is available at the end of the article
}

(c) The Author(s). 2021 Open Access This article is licensed under a Creative Commons Attribution 4.0 International License, which permits use, sharing, adaptation, distribution and reproduction in any medium or format, as long as you give appropriate credit to the original author(s) and the source, provide a link to the Creative Commons licence, and indicate if changes were made. The images or other third party material in this article are included in the article's Creative Commons licence, unless indicated otherwise in a credit line to the material. If material is not included in the article's Creative Commons licence and your intended use is not permitted by statutory regulation or exceeds the permitted use, you will need to obtain permission directly from the copyright holder. To view a copy of this licence, visit http://creativecommons.org/licenses/by/4.0/ The Creative Commons Public Domain Dedication waiver (http://creativecommons.org/publicdomain/zero/1.0/) applies to the data made available in this article, unless otherwise stated in a credit line to the data. 


\section{Background}

Lower respiratory tract infections are common in hospitalized patients, particularly among patients in intensive care units (ICUs), and are associated with significant morbidity and mortality [1]. Gram-negative bacilli, including Pseudomonas aeruginosa and Enterobacterales are frequent and important pathogens in patients with hospital-acquired and ventilator-associated pneumonia. $P$. aeruginosa is a particularly important nosocomial pathogen because it possesses intrinsic resistance to several antimicrobial agent classes and not uncommonly demonstrates resistance to antipseudomonal agents.

Ceftolozane/tazobactam $(\mathrm{C} / \mathrm{T})$ is a parenteral agent that combines an antipseudomonal cephalosporin with tazobactam, an established $\beta$-lactamase inhibitor of many Ambler class A $\beta$-lactamases (excluding KPC-type and some GES-type carbapenemases and the ESBL PER1) [2]. $\mathrm{C} / \mathrm{T}$ demonstrates potent in vitro activity against many clinically important Gram-negative bacilli and enhanced activity against $P$. aeruginosa [3-9]. C/T is approved in 70 countries, including the United States for the treatment of patients with hospital-acquired and ventilator-associated bacterial pneumonia ( 3 g every $8 \mathrm{~h}$ ), and in 75 countries for the treatment of complicated intraabdominal infection ( $1.5 \mathrm{~g}$ every $8 \mathrm{~h}$, used in combination with metronidazole), and complicated urinary tract infection $(1.5 \mathrm{~g}$ every $8 \mathrm{~h}$ ), including pyelonephritis [10]. $\mathrm{C} / \mathrm{T}$ is an important agent for the treatment of patients with severe infections due to multidrug-resistant (MDR) P. aeruginosa [11], which is a common phenotype in this pathogen in many countries, including the United States [4, 6, 12-14]. C/T may also provide an alternative to carbapenems for the treatment of infections caused by Gram-negative bacilli, including extendedspectrum $\beta$-lactamase (ESBL)-producing isolates [15-17].

The current report summarizes antimicrobial susceptibility testing results for $\mathrm{C} / \mathrm{T}$ for isolates of $P$. aeruginosa and Enterobacterales submitted to the SMART (Study for Monitoring Antimicrobial Resistance Trends) global surveillance program in 2018-2019 by clinical laboratories from the United States, focusing on isolates from lower respiratory tract infections.

\section{Results}

Isolates of $P$. aeruginosa were highly susceptible (96.0\%) to $\mathrm{C} / \mathrm{T}(\mathrm{MIC} \leq 4 \mu \mathrm{g} / \mathrm{ml}$ ) (Table 1$) ;<80 \%$ of isolates of $P$. aeruginosa were susceptible to the other $\beta$-lactams tested, including cefepime, ceftazidime, piperacillin/tazobactam $(\mathrm{P} / \mathrm{T})$, and meropenem. Susceptibility to amikacin $(96.0 \%)$ was identical to $\mathrm{C} / \mathrm{T}$ against $P$. aeruginosa. Most isolates of Enterobacterales (91.8\%) were also susceptible to $\mathrm{C} / \mathrm{T}(\mathrm{MIC} \leq 2 \mu \mathrm{g} / \mathrm{ml})$ (Table 1$)$. The susceptibility of isolates of Enterobacterales to $\mathrm{C} / \mathrm{T}$ was 2.3 (cefepime) to 11.7 (ceftriaxone) percentage points higher than all other $\beta$-lactams tested except meropenem (98.0\% susceptible). Amikacin was the most active agent tested against Enterobacterales (98.8\% susceptible). Against individual species of Enterobacterales, $\mathrm{C} / \mathrm{T}$ was most active against Proteus mirabilis (99.2\% susceptible), Klebsiella oxytoca (97.8\%), and Escherichia coli (97.2\%). The study identified $49(4.0 \%)$ isolates of $P$. aeruginosa and $158(8.2 \%)$ isolates of Enterobacterales (Table 1, Additional file 1: Table S1) that were C/T-nonsusceptible; $70 \%(110 / 158)$ of $\mathrm{C} / \mathrm{T}$-nonsusceptible Enterobacterales isolates were intrinsic AmpC producers. Table 2 summarizes the in vitro activities of $\mathrm{C} / \mathrm{T}$ and comparator

Table 1 Susceptibility of lower respiratory tract infection isolates of $P$. aeruginosa and Enterobacterales to C/T and comparator agents $^{\mathrm{a}, \mathrm{b}}-$ SMART 2018-2019, United States

\begin{tabular}{|c|c|c|c|c|c|c|c|c|c|c|c|}
\hline \multirow[t]{2}{*}{ Species } & \multirow[t]{2}{*}{$n$} & \multicolumn{10}{|c|}{$\%$ Susceptible } \\
\hline & & $\mathrm{C} / \mathrm{T}$ & $\mathrm{P} / \mathrm{T}$ & FEP & CAZ & CRO & ATM & MEM & IMI & LVX & $\overline{A M K}$ \\
\hline P. aeruginosa & 1237 & 96.0 & 74.4 & 79.2 & 78.5 & NA & 68.2 & 74.8 & 65.2 & 63.1 & 96.0 \\
\hline All Enterobacterales ${ }^{c}$ & 1938 & 91.8 & 88.0 & 89.5 & 83.4 & 80.1 & 84.2 & 98.0 & 86.4 & 82.2 & 98.8 \\
\hline K. pneumoniae & 432 & 92.6 & 86.8 & 82.2 & 79.9 & 80.3 & 81.9 & 96.5 & 95.1 & 81.0 & 98.4 \\
\hline E. coli & 359 & 97.2 & 91.9 & 84.7 & 82.2 & 79.4 & 82.5 & 99.7 & 99.7 & 66.6 & 98.9 \\
\hline S. marcescens & 261 & 95.8 & 93.5 & 96.6 & 95.8 & 84.3 & 93.5 & 96.9 & 84.3 & 90.8 & 97.3 \\
\hline E. cloacae & 176 & 77.8 & 79.0 & 89.8 & 72.2 & 69.3 & 73.9 & 98.9 & 94.3 & 94.3 & 100 \\
\hline K. aerogenes & 152 & 79.6 & 73.0 & 94.1 & 70.4 & 67.8 & 72.4 & 98.0 & 73.0 & 93.4 & 100 \\
\hline K. oxytoca & 138 & 97.8 & 87.7 & 94.9 & 96.4 & 87.0 & 91.3 & 97.8 & 97.1 & 99.3 & 100 \\
\hline P. mirabilis & 121 & 99.2 & 100 & 95.0 & 96.7 & 95.9 & 97.5 & 100 & 19.8 & 61.2 & 99.2 \\
\hline P. aeruginosa + Enterobacterales & 3175 & 93.5 & 82.7 & 85.5 & 81.5 & NA & 78.0 & 88.9 & 78.2 & 74.8 & 97.7 \\
\hline
\end{tabular}

${ }^{a}$ Abbreviations: C/T, ceftolozane/tazobactam; P/T, piperacillin/tazobactam; FEP, cefepime; CAZ, ceftazidime; CRO, ceftriaxone; ATM, aztreonam; MEM, meropenem; IMI, impenem; LVX, levofloxacin; AMK, amikacin, NA, not available

b Table does not show results for colistin because P. aeruginosa and Enterobacterales cannot be categorized as susceptible to colistin using CLSI M100 (2020) MIC breakpoint criteria

c Table only shows data for individual species of Enterobacterales when $n>100$ 
Table 2 Susceptibility of phenotypic subsets of lower respiratory tract infection isolates of $P$. aeruginosa and Enterobacterales to $C / T$ and comparator agents ${ }^{\mathrm{a}, \mathrm{b}}$ - SMART 2018-2019, United States

\begin{tabular}{|c|c|c|c|c|c|c|c|c|c|c|c|}
\hline \multirow[t]{2}{*}{ Species/phenotype } & \multirow[t]{2}{*}{$n$} & \multicolumn{10}{|c|}{ \% Susceptible } \\
\hline & & $\overline{C / T}$ & $\mathrm{P} / \mathrm{T}$ & FEP & CAZ & CRO & ATM & MEM & IMI & LVX & $\overline{A M K}$ \\
\hline \multicolumn{12}{|l|}{ P. aeruginosa } \\
\hline FEP-nonsusceptible & 257 & 82.1 & 12.5 & 0 & 18.3 & NA & 9.3 & 38.9 & 35.4 & 31.1 & 86.4 \\
\hline CAZ-nonsusceptible & 266 & 81.6 & 10.2 & 21.1 & 0 & NA & 12.8 & 46.6 & 41.0 & 38.4 & 88.7 \\
\hline MEM-nonsusceptible & 312 & 87.8 & 41.7 & 49.7 & 54.5 & NA & 31.1 & 0 & 7.1 & 30.1 & 90.1 \\
\hline P/T-nonsusceptible & 317 & 86.8 & 0 & 29.0 & 24.6 & NA & 12.3 & 42.6 & 40.4 & 36.0 & 91.2 \\
\hline MDR & 181 & 76.2 & 8.8 & 12.7 & 17.1 & NA & 3.9 & 17.7 & 15.5 & 17.7 & 82.3 \\
\hline \multicolumn{12}{|l|}{ Enterobacterales } \\
\hline FEP-nonsusceptible & 204 & 59.3 & 50.5 & 0 & 10.3 & 2.0 & 9.3 & 84.8 & 78.4 & 31.4 & 91.7 \\
\hline CAZ-nonsusceptible & 321 & 52.7 & 43.9 & 43.0 & 0 & 3.7 & 11.2 & 89.4 & 81.9 & 52.0 & 95.0 \\
\hline MEM-nonsusceptible & 39 & 12.8 & 10.3 & 20.5 & 12.8 & 7.7 & 7.7 & 0 & 0 & 38.5 & 84.6 \\
\hline P/T-nonsusceptible & 232 & 36.2 & 0 & 56.5 & 22.4 & 16.4 & 22.8 & 84.9 & 80.2 & 66.4 & 94.0 \\
\hline MDR & 238 & 49.6 & 42.9 & 30.7 & 8.8 & 7.1 & 9.2 & 84.9 & 73.1 & 36.6 & 92.4 \\
\hline \multicolumn{12}{|l|}{ K. pneumoniae ${ }^{c}$} \\
\hline FEP-nonsusceptible & 77 & 63.6 & 49.4 & 0 & 3.9 & 2.6 & 5.2 & 80.5 & 79.2 & 28.6 & 90.9 \\
\hline CAZ-nonsusceptible & 87 & 63.2 & 47.1 & 14.9 & 0 & 8.1 & 11.5 & 82.8 & 80.5 & 33.3 & 92.0 \\
\hline P/T-nonsusceptible & 57 & 45.6 & 0 & 31.6 & 19.3 & 29.8 & 31.6 & 73.7 & 70.2 & 47.4 & 87.7 \\
\hline ESBL screen-positive ${ }^{d}$ & 85 & 67.1 & 52.9 & 11.8 & 5.9 & 0 & 9.4 & 82.4 & 80.0 & 32.9 & 91.8 \\
\hline MDR & 66 & 59.1 & 45.5 & 1.5 & 0 & 0 & 0 & 77.3 & 75.8 & 25.8 & 89.4 \\
\hline \multicolumn{12}{|l|}{ E. colic } \\
\hline FEP-nonsusceptible & 55 & 87.3 & 80.0 & 0 & 12.7 & 0 & 12.7 & 98.2 & 98.2 & 14.6 & 92.7 \\
\hline CAZ-nonsusceptible & 64 & 84.4 & 75.0 & 25.0 & 0 & 3.1 & 6.3 & 98.4 & 98.4 & 29.7 & 95.3 \\
\hline P/T-nonsusceptible & 29 & 69.0 & 0 & 62.1 & 44.8 & 48.3 & 51.7 & 96.6 & 96.6 & 51.7 & 93.1 \\
\hline ESBL screen-positive ${ }^{d}$ & 74 & 86.5 & 79.7 & 25.7 & 16.2 & 0 & 16.2 & 98.7 & 98.7 & 25.7 & 94.6 \\
\hline MDR & 45 & 82.2 & 73.3 & 4.4 & 0 & 0 & 2.2 & 97.8 & 97.8 & 11.1 & 93.3 \\
\hline \multicolumn{12}{|c|}{ P. aeruginosa + Enterobacterales } \\
\hline FEP-nonsusceptible & 461 & 72.0 & 29.3 & 0 & 14.7 & NA & 9.3 & 59.2 & 54.4 & 31.2 & 88.7 \\
\hline CAZ-nonsusceptible & 587 & 65.8 & 28.6 & 33.0 & 0 & NA & 11.9 & 70.0 & 63.4 & 45.8 & 92.2 \\
\hline MEM-nonsusceptible & 351 & 79.5 & 38.2 & 46.4 & 49.9 & NA & 28.5 & 0 & 6.3 & 31.1 & 89.5 \\
\hline $\mathrm{P} / \mathrm{T}$-nonsusceptible & 549 & 65.4 & 0 & 40.6 & 23.7 & NA & 16.8 & 60.5 & 57.2 & 48.8 & 92.4 \\
\hline MDR & 419 & 61.1 & 28.2 & 22.9 & 12.4 & NA & 6.9 & 55.8 & 48.2 & 28.4 & 88.1 \\
\hline
\end{tabular}

${ }^{a}$ Abbreviations: C/T, ceftolozane/tazobactam; P/T, piperacillin/tazobactam; FEP, cefepime; CAZ, ceftazidime; CRO, ceftriaxone; ATM, aztreonam; MEM, meropenem; IMI, impenem; LVX, levofloxacin; AMK, amikacin; NA, not available

b Table does not show results for colistin because $P$. aeruginosa and Enterobacterales cannot be categorized as susceptible to colistin using CLSI M100 (2020) MIC breakpoint criteria

${ }^{c}$ MEM-nonsusceptible $E$. coli $(n=1)$ and MEM- nonsusceptible $K$. pneumoniae $(n=15)$ were not included in the table because there were too few isolates

${ }^{d}$ ESBL screen-positive isolates of $K$. pneumoniae and $E$. coli were identified by a CRO-nonsusceptible phenotype

agents against nonsusceptible subsets of isolates and MDR isolates of $P$. aeruginosa and Enterobacterales; $14.6 \%(181 / 1237)$ of $P$. aeruginosa and $12.3 \%$ (238/1938) of Enterobacterales possessed MDR phenotypes. Table S2 shows the 5 most commonly identified MDR phenotypes among $P$. aeruginosa and Enterobacterales (Additional file 2). $\mathrm{C} / \mathrm{T}$ inhibited $76.2 \%$ of all MDR isolates of $P$. aeruginosa (including $96.8 \%$ of isolates of the most common phenotype: resistant to aztreonam, imipenem, and levofloxacin $[n=31]$, Additional file 2: Table S2), and $>80 \%$ of cefepime-, ceftazidime-, meropenem-, or P/ T-nonsusceptible $P$. aeruginosa (Table 2). The susceptibility of MDR isolates of $P$. aeruginosa to $C / T$ was $58.5-$ 72.3 percentage points higher than to other $\beta$-lactams and levofloxacin. Only amikacin showed a higher rate of susceptibility than $\mathrm{C} / \mathrm{T}$ against MDR isolates of $P$. aeruginosa. $\mathrm{C} / \mathrm{T}$ inhibited $49.6 \%$ of MDR Enterobacterales (including $90.4 \%$ of isolates of the most common 
phenotype: resistant to aztreonam, cefepime, ceftazidime, and levofloxacin [ $n=52]$, Additional file 2: Table S2), and > $50 \%$ of cefepime- or ceftazidime-nonsusceptible Enterobacterales (Table 2). The susceptibility of MDR isolates of Enterobacterales to $\mathrm{C} / \mathrm{T}$ was $6.7(\mathrm{P} / \mathrm{T})$ to 42.4 (ceftriaxone) percentage points higher than to non-carbapenem $\beta$-lactam comparator antimicrobial agents and levofloxacin. Only amikacin and carbapenems showed higher susceptibility rates than $\mathrm{C} / \mathrm{T}$ against MDR isolates of Enterobacterales. 67.1 and $86.5 \%$ of ESBL screen-positive isolates of Klebsiella pneumoniae $(n=85)$ and Escherichia coli $(n=74)$ were susceptible to $\mathrm{C} / \mathrm{T}$ compared to 52.9 and $79.7 \%$, respectively, for $\mathrm{P} / \mathrm{T}$.

Table 3 summarizes the species distribution among Enterobacterales collected from patients with lower respiratory tract infections in ICU and non-ICU wards and shows very similar species distributions between the two ward types. Table 4 shows the susceptibility to various antimicrobial agents of $P$. aeruginosa and Enterobacterales from patients with lower respiratory tract infections in ICU and non-ICU wards. Percent susceptible rates were significantly lower for $\mathrm{P} / \mathrm{T}$, cefepime, ceftazidime, and meropenem for ICU ward isolates compared to non-ICU ward isolates for $P$. aeruginosa $(P<0.05)$; no significant differences between ICU and non-ICU ward isolates were identified for any agent tested against Enterobacterales $(P>0.05) . \quad \mathrm{C} / \mathrm{T}$ was equally active against ICU and non-ICU isolates of $P$. aeruginosa ( 96.0 and $96.7 \%$ susceptible, respectively) and of Enterobacterales (91.3 and 92.6\%, respectively).

\section{Discussion}

Empirical regimens to treat hospital-acquired and ventilator-associated pneumonia are based on the most prevalent pathogens causing infection and their antimicrobial susceptibility patterns. Prompt and

Table 3 Species distribution among Enterobacterales collected from patients with RTI in ICU $(n=1005)$ and non-ICU wards $(n=$ 726) - SMART 2018-2019, United States ${ }^{a}$

\begin{tabular}{lll}
\hline Species of Enterobacterales & $\begin{array}{l}\text { ICU, } \boldsymbol{n} \text { (\% of ICU } \\
\text { isolates of } \\
\text { Enterobacterales) }\end{array}$ & $\begin{array}{l}\text { Non-ICU, } \boldsymbol{n} \text { (\% of } \\
\text { non-ICU isolates of } \\
\text { Enterobacterales) }\end{array}$ \\
\hline K. pneumoniae & $234(23.3)$ & $156(21.5)$ \\
E. coli & $187(18.6)$ & $129(17.8)$ \\
S. marcescens & $130(12.9)$ & $102(14.0)$ \\
E. cloacae & $86(8.6)$ & $66(9.1)$ \\
K. aerogenes & $84(8.4)$ & $52(7.2)$ \\
K. oxytoca & $71(7.1)$ & $53(7.3)$ \\
P. mirabilis & $60(6.0)$ & $48(6.6)$ \\
Other species & $153(15.2)$ & $120(16.5)$ \\
\hline
\end{tabular}

${ }^{a}$ Of the 1938 isolates of Enterobacterales, 57 were from emergency rooms and 150 did not have a location provided and were excluded from this table appropriate antimicrobial treatment decreases infectionrelated morbidity and mortality; resistant bacteria lead to inadequate empirical treatment and are associated with patient mortality [1]. In the current study of lower respiratory tract infection isolates of Gram-negative bacilli, C/T was active against $96.0 \%$ of isolates of $P$. aeruginosa, $91.8 \%$ of isolates of Enterobacterales, and $93.5 \%$ of $P$. aeruginosa and Enterobacterales combined, which accounted for $>$ $85 \%$ of all collected Gram-negative pathogens (Table 1). $\mathrm{C} / \mathrm{T}$ has sustained its potent in vitro activity against $P$. aeruginosa and Enterobacterales with clinical use since it was first approved by the US FDA in 2014 and by EMA in $2015[7,8]$. C/T was approved for the treatment of adult patients with hospital-acquired and ventilator-associated bacterial pneumonia by the US FDA and EMA in 2019.

In the current study, $4.0 \%$ of isolates of $P$. aeruginosa and $8.2 \%$ isolates of Enterobacterales were $\mathrm{C} / \mathrm{T}$-nonsusceptible; $70 \%$ of the $\mathrm{C} / \mathrm{T}$-nonsusceptible Enterobacterales isolates were intrinsic $\mathrm{AmpC}$ producers. AmpC induction is an important $\beta$-lactam resistance mechanism. Different groups of $\beta$-lactams have different abilities to cause derepression of $\operatorname{ampC}[18,19]$. Ceftolozane is an extended-spectrum cephalosporin, and like other extended-spectrum cephalosporins (e.g., ceftazidime) is a poor inducer of $a m p C$ expression [18-20], making it an attractive treatment option for infections caused by $P$. aeruginosa and Enterobacterales. Ceftolozane is structurally similar to ceftazidime but possesses a pyrazole substituent at the 3-position side chain which provides steric hinderance and prevents or slows hydrolysis by most AmpC $\beta$-lactamases. Tazobactam is an irreversible inhibitor of some class $C \beta$-lactamases and a number of class A $\beta$-lactamases (most ESBLs, not KPCs). Tazobactam is generally a poor inducer of AmpC; however, when high expression levels of AmpC are present, tazobactam may be overwhelmed (similar to clavulanic acid and sulbactam), making it a poor inhibitor of AmpC. This may lead to resistance among Enterobacterales [21].

$\mathrm{C} / \mathrm{T}$ is highly active against $P$. aeruginosa which contain PDC (Pseudomonas derived cephalosporinase) a constitutive Ambler class $\mathrm{C} \beta$-lactamase (AmpC) with high sequence polymorphism (>300 sequence variants have been identified) [22, 23]. Recently, rare, individual isolates of $P$. aeruginosa that developed resistance to $\mathrm{C} /$ $\mathrm{T}$ and ceftazidime/avibactam during therapy by acquisition of structural mutations in, and over-expression of, PDC in a highly derepressed $a m p C$ background have been reported [24, 25].

Previous studies of $P$. aeruginosa have identified one or more of OprD loss, ampC derepression, or hyperexpression of efflux pumps as likely mechanisms leading to $\mathrm{P} / \mathrm{T}$, ceftazidime, cefepime, and carbapenem resistance $[9,22]$. Isolates of $P$. aeruginosa with carbapenem-resistant phenotypes generally do not carry carbapenemases $[22,26]$. 
Table 4 Susceptibility of ICU and non-ICU ward subsets of lower respiratory tract infection isolates of $P$. aeruginosa and Enterobacterales to C/T and comparator agents ${ }^{a, b, c}$ - SMART 2018-2019, United States

\begin{tabular}{|c|c|c|c|c|c|c|c|c|c|c|c|c|}
\hline \multirow{2}{*}{$\begin{array}{l}\text { Species/ward } \\
\text { type }\end{array}$} & \multirow[t]{2}{*}{$n$} & \multicolumn{10}{|c|}{$\%$ Susceptible } & \multirow[t]{2}{*}{$\%$ MDR } \\
\hline & & $\mathrm{C} / \mathrm{T}$ & $\mathrm{P} / \mathrm{T}$ & FEP & CAZ & CRO & ATM & MEM & IMI & LVX & AMK & \\
\hline \multicolumn{13}{|l|}{ P. aeruginosa ${ }^{d}$} \\
\hline ICU & 495 & 96.0 & 72.3 & 78.0 & 76.6 & NA & 68.1 & 72.9 & 64.4 & 66.7 & 96.2 & 17.8 \\
\hline Non-ICU & 583 & 96.7 & 79.4 & 83.0 & 82.9 & NA & 72.2 & 79.8 & 69.0 & 61.9 & 96.6 & 10.5 \\
\hline \multicolumn{13}{|c|}{ Enterobacterales $^{\mathrm{e}}$} \\
\hline $\mathrm{ICU}$ & 1005 & 91.3 & 87.4 & 89.4 & 83.2 & 80.5 & 83.3 & 97.5 & 87.5 & 81.7 & 99.0 & 13.0 \\
\hline Non-ICU & 726 & 92.6 & 89.3 & 89.4 & 83.3 & 79.6 & 85.1 & 98.5 & 85.1 & 83.0 & 98.6 & 11.7 \\
\hline \multicolumn{13}{|c|}{ P. aeruginosa + Enterobacterales } \\
\hline $\mathrm{ICU}$ & 1500 & 92.9 & 82.4 & 85.6 & 81.0 & NA & 78.3 & 89.4 & 79.9 & 76.7 & 98.1 & 14.6 \\
\hline Non-ICU & 1309 & 94.4 & 84.9 & 86.6 & 83.1 & NA & 79.4 & 90.1 & 77.9 & 73.6 & 97.7 & 11.2 \\
\hline
\end{tabular}

OprD porin loss and active efflux appear to have no impact on C/T MICs $[9,22,24]$. C/T is widely regarded as the most suitable option for the treatment of infections caused by non-carbapenemase-producing MDR P. aeruginosa [11].

The combination of tazobactam with ceftolozane protects ceftolozane from hydrolysis by the majority of ESBLproducing isolates of $E$. coli and K. pneumoniae; 90\% of ESBL-positive $E$. coli and $\sim 70 \%$ of ESBL-positive $K$. pneumoniae are susceptible to $\mathrm{C} / \mathrm{T}[4,5,7-9,17]$. Lower MICs in ESBL-producing E. coli $\left(\mathrm{MIC}_{50} / \mathrm{MIC}_{90}, 0.5 / 4 \mu \mathrm{g} / \mathrm{ml}\right)$ than in $K$. pneumoniae $\left(\mathrm{MIC}_{50} / \mathrm{MIC}_{90}, 4 />32 \mu \mathrm{g} / \mathrm{ml}\right)$ reflect differences in ESBL genes typically present in $E$. coli $\left(\right.$ bla $\left._{\text {CTX-M }}\right)$ and K. pneumoniae $\left(b l a_{\text {TEM/SHV }}\right)$ [4, 5, 7-9]. In the current study, $\mathrm{C} / \mathrm{T}$ demonstrated greater in vitro activity against all Enterobacterales and against ESBL screen-positive isolates than $\mathrm{P} / \mathrm{T}$ and currently available advanced-generation cephalosporins, as observed by previous investigators $[4,5,7-9,17]$.

Increased carbapenem use is observed in areas with greater prevalence of ESBL-producing Enterobacterales [15]. Carbapenems are frequently recommended for the treatment of ESBL-producing infections, even when isolates are susceptible in vitro to other $\beta$-lactams [15]. This practice as well as fluoroquinolone and ceftazidime use have contributed to the emergence and spread of carbapenemase-producing Enterobacterales, for which therapeutic options are limited. Adequately powered randomized controlled trials evaluating the use of noncarbapenem $\beta$-lactam agents for ESBL infections are needed to resolve conflicting results generated by retrospective and observational studies, which likely reflect the presence of confounding/uncontrolled factors [5, 15].
Whenever equally efficacious alternate agents exist, consideration should be given to limit the use of carbapenems in the treatment of potentially less serious infection types (e.g. urinary tract infections) caused by ESBL-producing Enterobacterales, preserving carbapenems for the treatment of serious infections to prolong their effectiveness. Timely carbapenem de-escalation may also reduce the clinical use of these agents [15]. C/T has also shown promise as a non-carbapenem option [17]. Reanalysis of data from a randomized controlled Phase 3 clinical trial data [17] suggested that $\mathrm{C} / \mathrm{T}$ may be an effective agent for treating patients with complicated urinary tract infection or complicated intraabdominal infection caused by ESBLproducing $E$. coli and $K$. pneumoniae, even when in vitro MICs for some patient isolates exceed the susceptible breakpoint of $\leq 2 \mu \mathrm{g} / \mathrm{mL}$ [17]. On the other hand, $\mathrm{C} / \mathrm{T}$ is ineffective against isolates carrying carbapenemases, including class A (KPC) and class D (OXA) $\beta$-lactamases. It is inactive against metallo- $\beta$-lactamases $(\mathrm{MBL})$, as are ceftazidime-avibactam, meropenem-vaborbactam, and imipenem/relebactam. The prevalence of all carbapenemases is low in the United States, but higher rates of serine carbapenemases and MBL have been reported in other geographic regions [27-30]. Local resistance patterns should be taken into account when making empiric therapy decisions.

MDR phenotypes are commonly observed among $P$. aeruginosa isolated from patients with lower respiratory tract infections in the United States $[4,6,12,13]$, including ICU patients [14]; $15-30 \%$ of unique patient isolates of $P$. aeruginosa demonstrated an MDR phenotype in these studies. Although different definitions of multidrug-resistance complicate comparisons of MDR 
rates across studies, the MDR rate in the current study, $14.6 \%$ of lower respiratory tract infection isolates of $P$. aeruginosa tested from U.S. patients, was comparable to the aforementioned studies. Among these MDR P. aeruginosa, $76.2 \%$ of isolates were susceptible to $\mathrm{C} / \mathrm{T}$ compared to $<20 \%$ susceptible to cefepime, ceftazidime, $\mathrm{P} / \mathrm{T}$, meropenem and levofloxacin (Table 2).

ICUs house vulnerable patients at high risk for infection. Gram-negative bacilli infecting patients in ICUs may be associated with elevated resistance rates and increased MDR [13]. In the current study, MDR rates were significantly higher and percent susceptible rates were significantly lower for $\mathrm{P} / \mathrm{T}$, cefepime, ceftazidime, and meropenem for ICU ward isolates compared to non-ICU ward isolates for $P$. aeruginosa $(P<0.05)$; no significant differences between ICU and non-ICU ward isolates were identified for any agent tested against Enterobacterales $(P>0.05)$. $\mathrm{C} / \mathrm{T}$ was active against $92.9 \%$ of isolates of $P$. aeruginosa and Enterobacterales combined from ICU patients (Table 4).

Isolates of Gram-negative bacilli that are not susceptible to first-line agents (i.e., all categories of $\beta$-lactams, including carbapenems, and fluoroquinolones) are associated with increased therapeutic failure and mortality especially in severely ill patients, many of whom reside within ICUs [31, 32]. Aminoglycosides (e.g., amikacin) and polymyxins (e.g., colistin) have toxicities (aminoglycosides, ototoxicity and nephrotoxicity; polymyxins, nephrotoxicity) that limit their use for serious Gramnegative infections and these agents are often used in combination with other antimicrobial agents [11, 31]. These agents also have suboptimal pharmacokinetics (e.g., poor lung penetration), a narrow therapeutic index and have demonstrated inferior efficacy to $\beta$-lactams as first-line therapies [11]. As of 2020, isolates of $P$. aeruginosa, Enterobacterales, and Acinetobacter can no longer test as susceptible in vitro to colistin using 2020 CLSI M100 MIC breakpoints [33] following review of clinical and pharmacokinetic/pharmacodynamic data demonstrating limited clinical efficacy of colistin. $\beta$-lactams are the safest class of antimicrobial agents available to treat patients with serious infection caused by Gram-negative bacilli.

\section{Conclusions}

We conclude that $\mathrm{C} / \mathrm{T}$ remains highly active against isolates of $P$. aeruginosa infecting patients in the United States, including isolates with MDR phenotypes. $\mathrm{C} / \mathrm{T}$ is an important treatment option for patients infected with $P$. aeruginosa isolates displaying an MDR phenotype that are not susceptible to other available $\beta$-lactam and fluoroquinolone anti-pseudomonal agents. Furthermore, $\mathrm{C} / \mathrm{T}$ can be a potential alternative for patients where colistin or an aminoglycoside is a therapeutic consideration, given the well-established toxicities associated with those agents and their poor lung penetration. $\mathrm{C} / \mathrm{T}$ was the most active $\beta$-lactam tested against $P$. aeruginosa. $\mathrm{C} / \mathrm{T}$ had in vitro activity similar to cefepime and $\mathrm{P} / \mathrm{T}$ against isolates of Enterobacterales, including putative ESBL-producing isolates of E. coli and K. pneumoniae. C/T was active against $93.5 \%$ of isolates of $P$. aeruginosa and Enterobacterales combined, which accounted for $>85 \%$ of all Gram-negative pathogens collected in the current study. Continuous surveillance monitoring for resistance is essential and provides critical data to guide empirical therapy, monitor the effectiveness of new antimicrobial agents and the emergence of resistance, and to monitor the performance of antimicrobial stewardship programs. Data from the current study support the use of $\mathrm{C} / \mathrm{T}$ as an important treatment option for lower respiratory tract infections caused by MDR P. aeruginosa and Enterobacterales.

\section{Methods \\ Bacterial isolates}

In 2018 and 2019, 24 clinical laboratories in 16 states in the United States participated in the Study for Monitoring Antimicrobial Resistance Trends (SMART) global surveillance program and were each asked to collect up to 100 clinically significant, consecutive, aerobic or facultatively anaerobic Gram-negative bacilli per year from patients with lower respiratory tract infection. Isolates were restricted to one isolate per patient per Gramnegative species per year. In total, 3717 isolates of Gram-negative bacilli were collected (Additional file 3: Fig. S1). The five most common species were $P$. aeruginosa (33.3\%), K. pneumoniae (11.6\%), E. coli (9.7\%), Stenotrophomonas maltophilia (7.3\%), and Serratia marcescens (7.0\%). The age distribution of patients from which isolates were collected was $0-17$ years $(n=468$, $12.6 \%), 18-64$ years $(n=1781,47.9 \%), \geq 65$ years $(1363$, $36.7 \%)$, and unknown age $(105,2.8 \%)$. P. aeruginosa $(n=$ $1237)$ and Enterobacterales $(n=1938)$ together accounted for $85.4 \%(3175 / 3717)$ of all isolates collected. Among P. aeruginosa and Enterobacterales, $50.5 \%$ of isolates $(1604 / 3175)$ were collected $\geq 48 \mathrm{~h}$ after the patient was admitted to the hospital, $34.8 \%(1104 / 3175)<48 \mathrm{~h}$ post-admission, and for $14.7 \%$ (467/3175) the length of stay was not specified. All isolates were transported to a central laboratory (IHMA, Schaumburg, IL, USA) where they were re-identified using matrix-assisted laser desorption ionization-time of flight (MALDI-TOF) mass spectrometry (Bruker Daltonics, Billerica, MA, USA).

Ethical approval and informed consent was not required because all isolates received into the study followed multiple subcultures and were completely deidentified. The secondary research use of de-identified isolates is considered exempt research according to the Regulations for the Protection of Human Subjects in 
Research of the U.S. Department of Health and Human Services, Office for Human Research Protections (45 CFR 46).

\section{Antimicrobial susceptibility testing}

MICs were determined using the CLSI (Clinical and Laboratory Standards Institute) reference broth microdilution method with frozen broth microdilution panels prepared at IHMA [33, 34]. MICs were interpreted using 2020 CLSI breakpoints [33]. MDR isolates were defined, phenotypically, as those testing resistant to three or more of the following eight sentinel antimicrobial agents: amikacin, aztreonam, cefepime, ceftazidime (Enterobacterales only), colistin, imipenem, levofloxacin, and P/T.

\section{Statistical analysis}

The Chi-square statistic with Yates correction (XLSTAT version 2019.1.3) was used to establish statistical significance $(P<0.05)$ between variables.

\section{Supplementary Information}

The online version contains supplementary material available at https://doi. org/10.1186/s12866-021-02135-z.

Additional file 1: Table S1. Species distribution of $C / T$-nonsusceptible Enterobacterales - SMART 2018-2019, United States

Additional file 2: Table S2. In vitro susceptibility to $\mathrm{C} / \mathrm{T}$ of the most common MDR phenotypes of $P$. aeruginosa and Enterobacterales

Additional file 3: Fig. S1. Species distribution of 3717 isolates of Gramnegative bacilli collected from patients with lower respiratory tract infections - SMART 2018-2019, United States

\section{Acknowledgements}

The authors thank all SMART participants for their contributions to the program.

\section{Authors'contributions}

JAK, SHL, KY, MRM, and DFS were involved in the design of the study. SHL and JAK performed the data analysis, JAK wrote the manuscript, and SHL and $K Y$ edited the manuscript. JAK, SHL, KY, MRM, and DFS read and approved the final manuscript.

\section{Funding}

Funding for this research, which included compensation for services related to preparing this manuscript, was provided by Merck Sharp \& Dohme Corp., a subsidiary of Merck \& Co., Inc., Kenilworth, NJ, USA. The sponsor participated in the development of the overall study design, but collection and testing of isolates, data analysis and manuscript preparation were independently performed by IHMA.

\section{Availability of data and materials}

Datasets used and analyzed for this study are available from the corresponding author upon reasonable request.

\section{Ethics approval and consent to participate}

Ethical approval and informed consent was not required because all isolates received into the study followed multiple subcultures and were completely de-identified. The secondary research use of de-identified isolates is considered exempt research according to the Regulations for the Protection of Human Subjects in Research of the U.S. Department of Health and Human Services, Office for Human Research Protections (45 CFR 46).
Consent for publication

Not applicable.

\section{Competing interests}

SHL and DFS work for IHMA, which receives funding from Merck Sharp \& Dohme Corp., a subsidiary of Merck \& Co., Inc., Kenilworth, NJ, USA for the SMART surveillance program. JAK is a consultant to IHMA, and an employee of the University of Manitoba and Shared Health Manitoba. KY and MRM are employees of Merck Sharp \& Dohme Corp., a subsidiary of Merck \& Co., Inc., Kenilworth, NJ, USA, and own stock and options in Merck \& Co., Inc., Kenilworth, NJ, USA. The IHMA authors do not have personal financial interests in the sponsor of this manuscript (Merck Sharp \& Dohme Corp., a subsidiary of Merck \& Co., Inc., Kenilworth, NJ, USA).

\section{Author details}

${ }^{1}$ IHMA, 2122 Palmer Drive, Schaumburg, IL 60173, USA. ²Department of Medical Microbiology and Infectious Diseases, Max Rady College of Medicine, University of Manitoba, Winnipeg, MB R3E 0J9, Canada. ${ }^{3}$ Merck \& Co., Inc., Kenilworth, NJ 07033, USA.

Received: 20 November 2020 Accepted: 19 February 2021

Published online: 06 March 2021

\section{References}

1. de la Rosa JM O, Nordmann P, Poirel L. ESBLs and resistance to ceftazidime/ avibactam and ceftolozane/tazobactam combinations in Escherichia coli and Pseudomonas aeruginosa. J Antimicrob Chemother. 2019;74:1934-9.

2. Kalil AC, Metersky ML, Klompas M, Muscedere J, Sweeney DA, Palmer LB, et al. Management of Adults with Hospital-acquired and Ventilatorassociated Pneumonia: 2016 clinical practice guidelines by the Infectious Diseases Society of America and the American Thoracic Society. Clin Infect Dis. 2016:63:e61-111.

3. Schmidt-Malan S, Mishra AJ, Mushtaq A, Brinkman CL, Patel R. In vitro activity of imipenem-relebactam and ceftolozane-tazobactam against resistant Gram-negative bacilli. Antimicrob Agents Chemother. 2018;62: e00533-18.

4. Sader HS, Flamm RK, Carvalhaes CG, Castanheira M. Comparison of ceftazidime-avibactam and ceftolozane-tazobactam in vitro activities when tested against Gram-negative bacteria isolated from patients hospitalized with pneumonia in United States medical centers (2017-2018). Diagn Microbiol Infect Dis. 2020;96:114833.

5. Karlowsky JA, Kazmierczak KM, Young K, Motyl MR, Sahm DF. In vitro activity of ceftolozane/tazobactam against phenotypically defined extendedspectrum $\beta$-lactamase (ESBL)-positive isolates of Escherichia coli and Klebsiella pneumoniae isolated from hospitalized patients (SMART 2016). Diagn Microbiol Infect Dis. 2020;96:114925.

6. Shortridge D, Pfaller MA, Streit JM, Flamm RK. Antimicrobial activity of ceftolozane/tazobactam tested against contemporary (2015-2017) Pseudomonas aeruginosa isolates from a global surveillance programme. J Glob Antimicrob Resist. 2020;21:60-4.

7. Farrell DJ, Sader HS, Flamm RK, Jones RN. Ceftolozane/tazobactam activity tested against Gram-negative bacterial isolates from hospitalised patients with pneumonia in US and European medical centres (2012). Int J Antimicrob Agents. 2014:43:533-9.

8. Castanheira M, Duncan LR, Mendes RE, Sader HS, Shortridge D. Activity of ceftolozane-tazobactam against Pseudomonas aeruginosa and Enterobacteriaceae isolates collected from respiratory tract specimens of hospitalized patients in the United States during 2013 to 2015. Antimicrob Agents Chemother. 2018;62:e02125-17.

9. Livermore DM, Mushtaq S, Meunier D, Hopkins KL, Hill R, Adkin R, et al. Activity of ceftolozane/tazobactam against surveillance and 'problem' Enterobacteriaceae, Pseudomonas aeruginosa and non-fermenters from the British Isles. J Antimicrob Chemother. 2017;72:2278-89.

10. ZERBAXA (ceftolozane/tazobactam) for injection, for intravenous use. Package insert. Merck sharp \& Dohme Corp., Whitehouse Station, NJ, USA, 2019.

11. Pogue JM, Kaye KS, Veve MP, Patel TS, Gerlach AT, Davis SL, et al. Ceftolozane/tazobactam vs polymyxin or aminoglycoside-based regimens for the treatment of drug-resistant Pseudomonas aeruginosa. Clin Infect Dis. 2020;71:304-10. 
12. Sader HS, Huband MD, Castanheira M, Flamm RK. Pseudomonas aeruginosa antimicrobial susceptibility results from four years (2012 to 2015) of the international network for optimal resistance monitoring program in the United States. Antimicrob Agents Chemother. 2017;61:e02252-16.

13. Lob SH, Hoban DJ, Young K, Motyl MR, Sahm DF. Activity of imipenem/ relebactam against Gram-negative bacilli from global ICU and non-ICU wards: SMART 2015-2016. J Glob Antimicrob Resist. 2018;15:12-9.

14. Trinh TD, Zasowski EJ, Claeys KC, Lagnf AM, Kidambi S, Davis SL, Rybak MJ. Multidrug-resistant Pseudomonas aeruginosa lower respiratory tract infections in the intensive care unit: prevalence and risk factors. Diagn Microbiol Infect Dis. 2017;89:61-6.

15. Tamma PD, Rodriguez-Baňo J. The use of noncarbapenem $\beta$-lactams for the treatment of extended-spectrum $\beta$-lactamase infections. Clin Infect Dis. 2017;64:972-80.

16. Bassetti M, Vena A, Giacobbe DR, Falcone M, Tiseo G, Giannella M, et al. Ceftolozane/tazobactam for treatment of severe ESBL-producing Enterobacterales infections: a multicenter nationwide clinical experience (CEFTABUSE II study). Open Forum Infect Dis. 2020. https://doi.org/10.1093/ ofid/ofaa139.

17. Popejoy MW, Paterson DL, Cloutier D, Huntington JA, Miller B, Bliss CA, et al. Efficacy of ceftolozane/tazobactam against urinary tract and intra-abdominal infections caused by ESBL-producing Escherichia coli and Klebsiella pneumoniae: a pooled analysis of phase 3 clinical trials. J Antimicrob Chemother. 2017;72:268-72.

18. Tamma PD, Doi Y, Bonomo RA, et al. A primer on AmpC $\beta$-lactamases: necessary knowledge for an increasingly multidrug-resistant world. Clin Infect Dis. 2019;69:1446-55.

19. Jacoby GA. AmpC $\beta$-Lactamases. Clin Microbiol Rev. 2009;22:161-82.

20. Zhanel GG, Chung P, Adam H, et al. Ceftolozane/tazobactam: a novel cephalosporin/beta-lactamase inhibitor combination with activity against multidrug-resistant Gram-negative bacilli. Drugs. 2014;74:31-51.

21. Bebrone C, Lassaux P, Vercheval L, Sohier J, Jehaes A, Sauvage E, Galleni M. Current challenges in antimicrobial chemotherapy: focus on beta-lactamase inhibition. Drugs. 2010;70:651-79.

22. Castanheira M, Mills JC, Farrell DJ, Jones RN. Mutation-driven $\beta$-lactam resistance mechanisms among contemporary ceftazidime-non-susceptible Pseudomonas aeruginosa isolates from U.S. hospitals. Antimicrob Agents Chemother. 2014;58:6844-50.

23. Rodríguez-Martínez J-M, Poirel L, Nordmann P. Extended-spectrum cephalosporinases in Pseudomonas aeruginosa. Antimicrob Agents Chemother. 2009;53:1766-71.

24. Arca-Suárez J, Vázquez-Ucha JC, Fraile-Ribot PA, Lence E, Cabot G, MartínezGuitián M, Lasarte-Monterrubio C, et al. Molecular and biochemical insights into the in vivo evolution of AmpC-mediated resistance to ceftolozane/ tazobactam during treatment of an MDR Pseudomonas aeruginosa infection. J Antimicrob Chemother. 2020. https://doi.org/10.1093/jac/dkaa291.

25. Fraile-Ribot PA, Cabot $G$, Mulet $X$, Periañez L, Martín-Pena ML, Juan C, et al. Mechanisms leading to in vivo ceftolozane/tazobactam resistance development during the treatment of infections caused by MDR Pseudomonas aeruginosa. J Antimicrob Chemother. 2018;73:658-63.

26. Kazmierczak KM, Rabine S, Hackel M, McLaughlin RE, Biedenbach DJ, Bouchillon SK, Sahm DF, Bradford PA. Multiyear, multinational survey of the incidence and global distribution of metallo- $\beta$-lactamase-producing Enterobacteriaceae and Pseudomonas aeruginosa. Antimicrob Agents Chemother. 2016;60:1067-78.

27. Bonomo RA, Burd EM, Conly J, Limbago BM, Poirel L, Segre JA, et al Carbapenemase-producing organisms: a global scourge. Clinical infectious diseases. 2018:66:1290-7.

28. Pitout JDD, Peirano G, Kock MM, Strydom K-A, Matsumura Y. The global ascendency of OXA-48-type Carbapenemases. Clin Microbiol Rev. 2019;33: e00102-19.

29. van Duin D, Doi Y. The global epidemiology of carbapenemase-producing Enterobacteriaceae. Virulence. 2017;8:460-9.

30. Boyd SE, Livermore DM, Hooper DC, Hope WW. Metallo- $\beta$-lactamases: structure, function, epidemiology, treatment options, and the development pipeline. Antimicrob Agents Chemother. 2020;64:e00397-20.

31. Kadri SS, Adjemian J, Lai YL, Spaulding AB, Ricotta E, Prevots DR, Palmore TN, et al. Difficult-to-treat resistance in Gram-negative bacteremia at 173 US hospitals: retrospective cohort analysis of prevalence, predictors, and outcome of resistance to all first-line agents. Clin Infect Dis. 2018;67:1803-14.
32. Huh K, Chung DR, Ha YE, Ko J-H, Kim S-H, Kim M-J, et al. Impact of difficultto-treat resistance in Gram-negative bacteremia on mortality: retrospective analysis of nationwide surveillance data. Clin Infect Dis 2020; doi/https://doi. org/10.1093/cid/ciaa084/5717239.

33. Clinical and Laboratory Standards Institute. Performance standards for antimicrobial susceptibility testing. 2020. 30th edition, M100. Clinical and laboratory standards institute, Wayne, PA, USA

34. Clinical and Laboratory Standards Institute. Methods for dilution antimicrobial susceptibility tests for bacteria that grow aerobically. 2018. 11th edition, M07-A11. Approved standard. Clinical and Laboratory Standards Institute, Wayne, PA, USA.

\section{Publisher's Note}

Springer Nature remains neutral with regard to jurisdictional claims in published maps and institutional affiliations.
Ready to submit your research? Choose BMC and benefit from:

- fast, convenient online submission

- thorough peer review by experienced researchers in your field

- rapid publication on acceptance

- support for research data, including large and complex data types

- gold Open Access which fosters wider collaboration and increased citations

- maximum visibility for your research: over $100 \mathrm{M}$ website views per year

At BMC, research is always in progress.

Learn more biomedcentral.com/submissions 